\title{
Analysis of high frequency guided wave scattering at a fastener hole with a view to fatigue crack detection
}

\author{
Bernard Masserey ${ }^{\text {a) }}$ \\ Department of Mechanical Engineering, University of Applied Sciences, \\ 1705 Fribourg, Switzerland \\ and \\ Paul Fromme b) \\ Department of Mechanical Engineering, University College London \\ London WC1E 7JE, United Kingdom
}

High frequency guided wave scattering

\footnotetext{
a) Electronic mail: bernard.masserey@ hefr.ch

b) Electronic mail: p.fromme@ucl.ac.uk
} 


\section{Abstract}

2 The scattering of high frequency guided ultrasonic waves by a fatigue crack at the side of a

3 fastener hole has been studied. The guided wave pulse consists of the superposition of the two

4 fundamental Lamb modes $\mathrm{A}_{0}$ and $\mathrm{S}_{0}$ above the cut-off frequencies of the higher modes. The

5 scattered field was simulated using a three-dimensional finite difference algorithm with a

6 staggered, Cartesian grid for the limited area of interest around the hole and an analytical phase

7 angle correction for the additional, variable propagation distance. Experimentally, the modes

8 were selectively excited using a standard ultrasonic wedge transducer and measured using a

9 laser interferometer, resulting in good spatial resolution. The scattered field was measured and

10 simulated for an undamaged hole, a small, part-thickness quarter-elliptical fatigue crack, and a

11 through-thickness fatigue crack. Good agreement was found and a significant influence of the

12 fatigue cracks on the scattered field was observed. The complex difference of the scattered

13 field due to additional scattered waves at the fatigue cracks of variable depth and length was

14 evaluated. This allows for the sensitivity prediction of high frequency guided waves for fatigue

15 crack detection at fastener holes, a significant maintenance problem for ageing aircraft.

16

17 Keywords: Lamb Waves, Scattering, Laser Measurement, Finite Difference Method 


\section{Introduction}

2 This contribution investigates the scattering of high frequency guided waves at fastener holes

3 with a view towards the non-destructive fatigue crack detection for aircraft and other technical

4 structures. For the aircraft industry a requirement exists for the nondestructive inspection and

5 monitoring of these structures to detect fatigue cracks before they have reached a critical length

6 and different nondestructive methods and sensors have been developed [1]. Ultrasonic bulk

7 waves have the required sensitivity for the detection and sizing of cracks [2] and can be

8 employed for in-situ monitoring of fastener hole cracks using an angle beam through

9 transmission technique [3]. Guided waves below the cutoff frequencies of the higher Lamb

10 modes are often used for large structures, as they can propagate long distances and allow for

11 the rapid and cost-efficient inspection and permanent monitoring of large surface areas [4]

12 without the requirement for local access. The scattering at holes in a plate was studied

13 analytically for flexural waves ( $A_{0}$ mode) [5, 6], and for the $S_{0}$ mode [7]. The scattered field of

14 the $\mathrm{A}_{0}$ Lamb wave mode at a hole with a crack was compared to finite difference simulations

15 [8]. Guided waves were found to successfully detect defects at difficult to reach locations

16 around a hole [9] and fatigue cracks emanating at fasteners within a lap joint [10].

17 As the ratio of the wavelength to the defect size determines the detection sensitivity, high

18 frequency guided waves have been investigated to improve the detection of small fatigue cracks

19 as compared to lower frequency guided waves [11]. Finite element simulations combined with modal decomposition were used to study the interaction of Lamb waves with defects for

21 frequency-thickness products up to $5 \mathrm{MHz} \mathrm{mm}$ [12]. High frequency Lamb waves at about 15

$22 \mathrm{MHz} \mathrm{mm}$ and the application for crack detection was demonstrated [13]. The weakly coupled

23 propagation of the two fundamental Lamb wave modes was assessed experimentally and the

24 resulting beatlength measurement compared to theoretical predictions [14]. This effect, described theoretically as a continuous energy exchange between the plate sides as the two modes propagate with slightly different phase velocities, is governed by the difference in the 
1 wave numbers of the two modes [15]. The significant distance $L$ for this, the so-called

2 beatlength, can be calculated as

$$
L=\frac{2 \pi}{k_{A 0}-k_{S 0}}
$$

where $k_{A 0}$ and $k_{S 0}$ are the wavenumbers of the $\mathrm{A}_{0}$ and $\mathrm{S}_{0}$ modes, respectively. For large

5 frequency-thickness products (small wavelength compared to the plate thickness), the

6 beatlength tends toward infinity and the wave propagation corresponds to a Rayleigh wave.

7 Different numerical methods were proposed to predict wave propagation and scattering in

8 linear elastic media. Hybrid Finite Element (FE) methods were used to study the scattering of

9 guided waves at a hole [16] and to compare to experiments for the scattering of the fundamental

$10 \mathrm{~S}_{0}$ mode at a hole with a crack [17]. Hybrid boundary methods were used to study Lamb wave

11 reflections and mode conversion at a plate edge above the lowest cut-off frequency [18]. Finite

12 Difference (FD) simulations based on a displacement formulation of the wave equations in

13 isotropic, linear elastic media were used to study the scattering of Lamb waves [19] and

14 Rayleigh waves [20] at cracks. Virieux [21] presented a two-dimensional, stress-velocity FD

15 formulation for modelling bulk wave propagation in heterogeneous media. The formulation

16 was based on a system of first-order hyperbolic equations discretized on a so-called staggered

17 grid. This type of grid, first proposed by Madariaga [22], has the useful property to minimize

18 the number of variables per grid cell. A displacement-velocity FD formulation was used to

19 investigate the two-dimensional near-field scattering of a Rayleigh wave at a surface breaking

20 crack with a view to the development of inversion schemes for crack sizing [23]. A comparable

21 formulation was used to investigate surface wave scattering in component-like specimens with complex geometries, where the specimen contours were approximated using a Cartesian grid

23 [24]. The stability criteria for this type of numerical simulations for the prediction of elastic

24 wave propagation and scattering in linear elastic media was discussed [25]. 
1 This contribution investigates the scattering of the coupled fundamental Lamb wave modes at

2 a circular hole with fatigue cracks. Experimentally the high frequency guided waves were

3 excited using standard angle beam transducers and the scattered field around the hole was

4 measured using laser interferometry. Full three-dimensional simulations of the scattering at the

5 fastener hole with crack were performed using a displacement-stress finite difference

6 formulation. The comparison of the results with measurements shows a good correlation of the

7 changes observed in the scattered field due to small part- and through-thickness fatigue cracks.

8 The complex difference of the scattered field due to fatigue cracks was analyzed.

\section{2. Experiments}

\section{$10 \quad$ 2.1. Specimens}

11 The $3 \mathrm{~mm}$ thick specimens were made from an aluminum alloy (2014 T6) widely used for 12 aerospace applications (Young's modulus $E$ of $73 \mathrm{GPa}$, density $\rho$ of $2800 \mathrm{~kg} / \mathrm{m}^{3}$, Poisson's

13 ratio $v$ of 0.33 ). The specimens were $600 \mathrm{~mm}$ long for the tensile cyclic loading and $70 \mathrm{~mm}$ 14 wide, so that the sides did not affect the propagation of the incident wave field. The fastener 15 hole was machined as a $1 / 4$ inch diameter hole $\left(r_{0}=3.17 \mathrm{~mm}\right)$ drilled on the center line at 200 $16 \mathrm{~mm}$ from the specimen end. The specimens investigated were either undamaged, had a small 17 (1.2 mm length $\times 1.2 \mathrm{~mm}$ depth) quarter-elliptical part-thickness fatigue crack, or a through thickness fatigue crack (4 mm length) at the side of the fastener hole as shown in Fig. 1. This length includes a small triangular starter notch, approximately $0.2 \mathrm{~mm}$ long, which was made in order to prescribe the fatigue crack initiation location. The fatigue cracks were generated using cyclic tensile loading and the crack size was measured optically using a microscope. The specimen was held under the maximum tensile load during the wave scattering measurements to avoid crack closure [11]. 


\section{2.2. Scattering measurements}

2 The high frequency guided wave pulse was excited using a standard $90^{\circ}$ angle beam transducer 3 and wedge, with a center frequency $f_{0}$ of $2.25 \mathrm{MHz}$ (Rayleigh wavelength in aluminum at 2.25

4 MHz: $\lambda_{R}=1.3 \mathrm{~mm}$ ). This excites primarily the two fundamental Lamb wave modes $\left(\mathrm{A}_{0}\right.$ and $5 \mathrm{~S}_{0}$ ) required for this investigation with approximately the same amplitudes. The first higher 6 antisymmetric Lamb mode $\left(\mathrm{A}_{1}\right)$ was found to be excited as well, but with approximately $20 \mathrm{~dB}$ 7 lower amplitude [26]. Due to its lower group velocity it arrives significantly after the

8 fundamental modes and could be time-gated out. Normal ultrasonic couplant was used and the

9 wedge was clamped to the tensile specimen at a distance $d=125 \mathrm{~mm}$ from the hole center on

10 the specimen surface opposite to the front surface where the starter notch was made (Fig. 1).

11 The distance, corresponding approximately to half a beatlength at the center frequency, was

12 selected in order to concentrate the energy on the specimen side containing the part-thickness

13 quarter-elliptical crack. The transducer was positioned in such a way that the main propagation

14 line crossed the center of the hole.

15

16

17

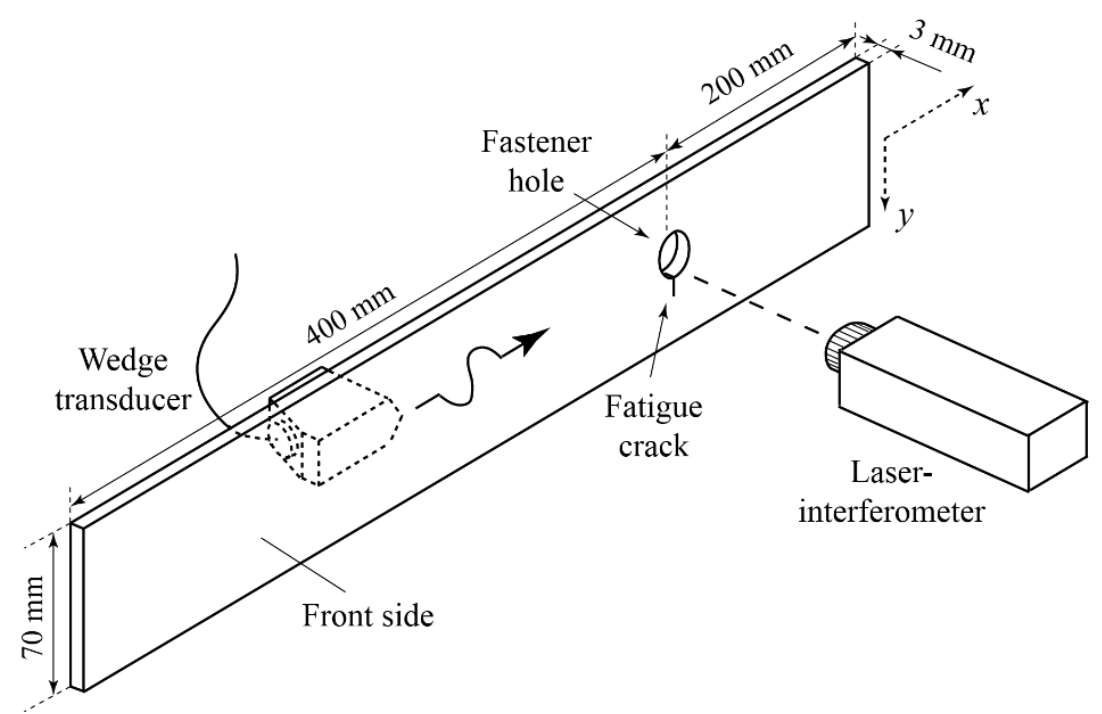

Fig 1: Schematic representation of specimen with wedge transducer and laser measurement close to fastener hole. 
1 The excitation was performed using specialized equipment, in order to achieve good control of

2 the frequency content of the excited pulse [27]. A 5-cycle tone burst (sinusoid multiplied by a

3 Hanning window) was generated in a programmable function generator and amplified using a

4 broadband power amplifier. The out-of-plane displacement close to the hole was measured

5 point-wise using a commercially available heterodyne laser interferometer [28]. The

6 measurement spot is defined by the laser beam diameter, which is well below $0.1 \mathrm{~mm}$, allowing

7 a detailed measurement of variations in the scattered field. The interferometer head was moved

8 parallel to the specimen surface using a scanning rig on a radial grid consisting of concentric

9 circles around the hole. An area from 3.3 to $8 \mathrm{~mm}$ radius around the fastener hole was covered

10 with a radial step size of $0.1 \mathrm{~mm}$ or $0.25 \mathrm{~mm}$ and an angular increment of 3 degrees. The

11 received signals were band pass filtered (1-5 MHz), averaged (100 averages), and recorded at

12 a sampling frequency of $100 \mathrm{MHz}$ using a digital storage oscilloscope. At each point of the

13 grid a time series with 5000 values was stored. A time gating was applied to cut off the later

14 arriving $A_{1}$ wave mode and reflections from the specimen boundaries, which contain no

15 information about the scattering at the hole. The time trace for each grid point was converted

16 to the frequency domain using Fast Fourier Transform (FFT) and the complex amplitude (phase

17 and amplitude) was extracted at the center frequency $f_{0}$. The scattered field was normalized on

18 the basis of the amplitude of the incident high frequency guided wave at center frequency, at a

19 position located on the central axis $(y=0) 8 \mathrm{~mm}$ before the hole center on the front side of the

20 specimen. Measurements were made on both the front (crack) and back (transducer) side of the 21 specimen.

\section{Numerical simulation}

\subsection{Finite difference formulation}

24 The propagation and scattering of the high frequency guided wave at the fastener hole was simulated using a three-dimensional (3D) second-order, displacement-stress Finite Difference 
1 (FD) formulation. For an isotropic, linear-elastic medium, the wave propagation is governed

2 by the equation of motion under small-deformation approximation,

$$
\sigma_{i j, j}=\rho \ddot{u}_{i},
$$

and the constitutive relation between stress and strain,

5

$$
\sigma_{i j}=\lambda \varepsilon_{k k} \delta_{i j}+2 \mu \varepsilon_{i j}
$$

6 where $\rho$ is the material density and the variables $\lambda$ and $\mu$ are known as Lamé's constants. The

$7 \quad$ indices $i, j$ and $k$ obey the index notation conventions and $\delta$ is the Kronecker delta symbol.

8 After insertion of the kinematic relation between strain tensor and displacement, the

9 constitutive equation can be written as :

$$
\sigma_{i j}=\lambda u_{k, k} \delta_{i j}+\mu\left(u_{i, j}+u_{j, i}\right)
$$

11 The governing equations (2) and (4) are discretized on a three dimensional Cartesian, staggered 12 grid with cubic cells [21]. This type of grid combines several types of nodes located at different 13 positions, allowing for a natural discretization of the governing equations while minimizing the 14 number of variables per grid cell. The corresponding unit cell of size $\Delta x, \Delta y$ and $\Delta z$ is shown 15 in Fig. 2. The displacement components, displayed by vectors in Fig. 2, are calculated in the 16 middle of the cell faces. The shear stresses, illustrated by circles for $\sigma_{x y}$, squares for $\sigma_{x z}$ and 17 triangles for $\sigma_{y z}$, are in the middle of the cell edges, and the center node, displayed by a black, 18 solid circle, corresponds to the three normal stress components. The through-thickness 19 discretization was applied in such a way that the out-of-plane displacement component $u_{z}$ and 20 the shear stress components $\sigma_{x z}$ and $\sigma_{y z}$ (see Fig. 2) are calculated on the front and back 21 surfaces of the plate. 


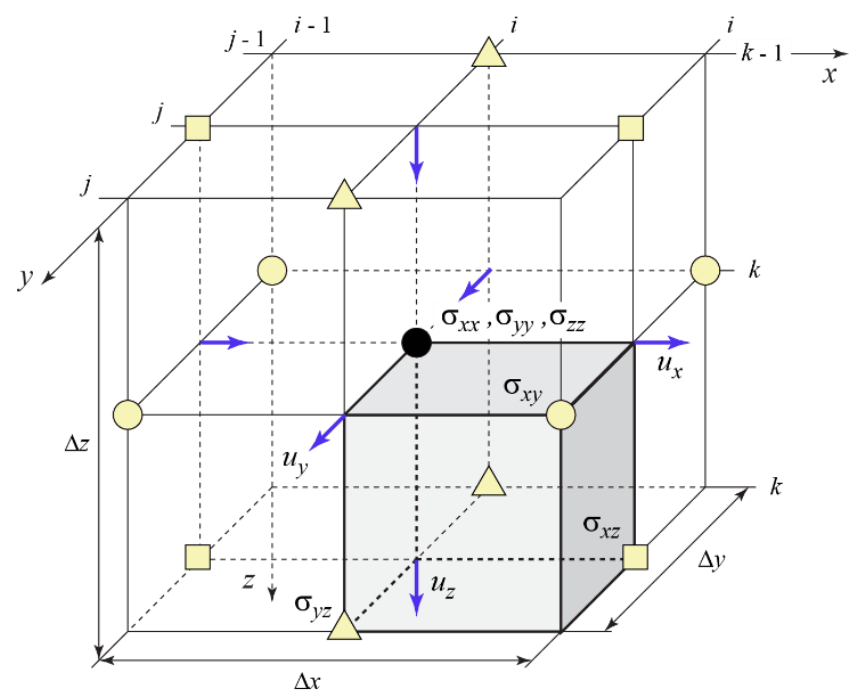

2 Fig. 2: Unit cell of displacement-stress finite difference formulation with normal stress node $x=i, y=j$ and $z=$

$3 \quad k$ (displayed by solid circle) in cell center.

4

5 The fastener hole (in the $x-y$ plane of the plate) is approximated with the Cartesian grid, the 6 grid size of $50 \mu \mathrm{m}$ being small compared to the $6.35 \mathrm{~mm}$ diameter hole. The approximated, 7 right-angled hole contour is chosen such that the corner points contain the nodes with the shear 8 stress component $\sigma_{x y}$ as illustrated schematically in Fig. 3a. Consequently, the shear stress 9 components $\sigma_{x z}$ and $\sigma_{y z}$ are located on the right-angled faces of the fastener hole with normal vector in the $x$ and $y$ directions, respectively. A shear stress value of zero is imposed for all 11 shear stress components located on the hole contour to fulfill the stress-free inner hole surface condition. As the nodal points containing the normal stress components are located half a cell inside the material (solid circles in Fig. 3a), fictitious normal stress nodes are added within the

14 hole half a cell from the right-angled boundary line (dotted circles in Fig. $3 a$ ). The normal

15 stress values $\sigma_{x x}$ and $\sigma_{y y}$ for the fictitious nodes are assigned with the same amplitude but 16 with opposite sign as the respective nodes within the material in $x$ - and $y$-directions, 17 respectively, in order to obtain a linearly interpolated value of zero at the stress-free Cartesian contour. 


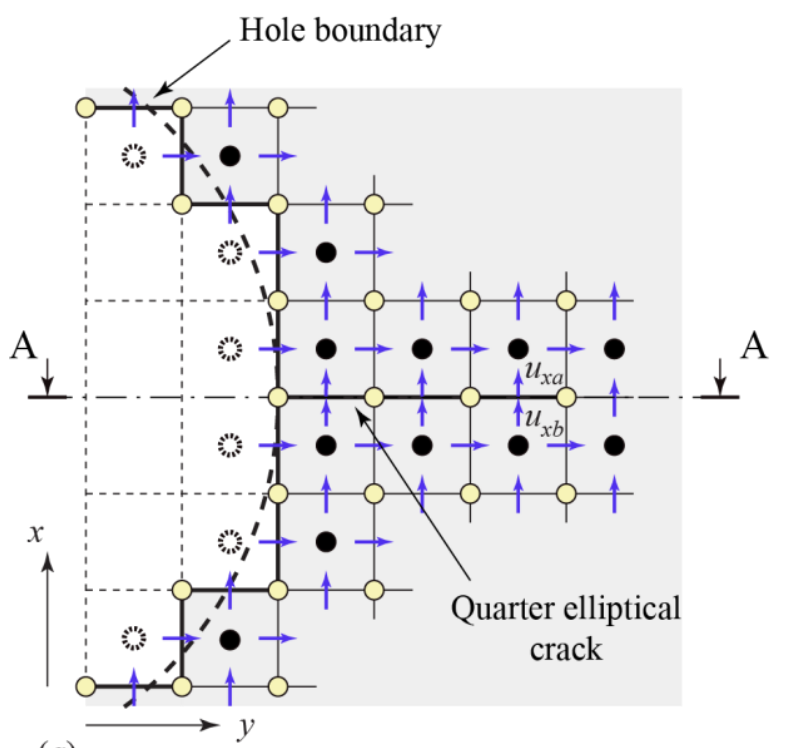

(a)

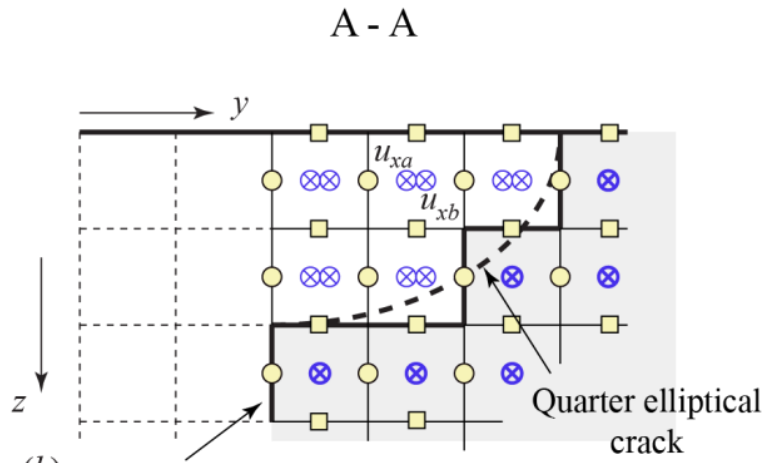

(b) Hole boundary

2 Fig. 3: Right-angled contour approximation and Cartesian grid for fastener hole with quarter elliptical crack.

3 View on specimen upper surface (a) and cross-section view in crack plane (b). Large grid size compared to hole radius and crack size selected for visualization purpose only. Symbols: vectors for displacement, solid circles for normal stress, circles for $\sigma_{x y}$, squares for $\sigma_{x z}$ and dashed circles for fictitious normal stress.

7 The fatigue crack is implemented in the $y-z$ plane at the side of the fastener hole as a quarterelliptical crack with zero width and variable length and depth, see Fig. $3 b$, or as a through thickness crack with zero width and the same length on both specimen surfaces. The crack is assumed to be open with stress-free inner crack faces and no interaction between the two faces.

11 As the crack faces and the crack contour are delimited by nodal points corresponding to the shear stress components $\sigma_{x y}$ and $\sigma_{x z}$, the corresponding values are set equal to zero during the simulation. In order to avoid the introduction of two additional fictitious layers for the normal stress component $\sigma_{x x}$, the displacement component in $x$-direction is split into two components $u_{x b}$ and $u_{x a}$ for the two sides of the crack, located on the same nodal points, see Fig. 3, but independent from each other. The displacement components $u_{x b}$ and $u_{x a}$ are evaluated using

17 the equation of motion in the $x$-direction and taking into account the stress-free boundary condition at the crack faces for the normal stress component $\sigma_{x x}$. The corresponding equations 
1 are based on the equations proposed by Masserey and Mazza [23] for two-dimensional

2 scattering of Rayleigh waves at a surface crack .

\section{3.2. Model size optimization based on mode separation}

4 As a general rule a minimum of 10 nodes per wavelength is required in order to reduce the so-

5 called numerical dispersion to an acceptable level [22]. For high-frequency guided waves, the

6 mode shapes through the specimen thickness have to be approximated accurately and in general

7 finer spatial sampling is required [23]. In this contribution, a grid size of $50 \mu \mathrm{m}$ was selected,

8 corresponding to a spatial sampling of 26 nodes per Rayleigh wavelength $(1.3 \mathrm{~mm}$ at 2.25

$9 \mathrm{MHz}$ ). As the implementation of the entire specimen in the FD formulation would result in

10 matrices with approximately 1000 million nodes and large computational requirements, the

11 specimen section considered in the simulation was reduced to a specimen length of $60 \mathrm{~mm}$

12 incorporating the fastener hole, keeping the full specimen width of $70 \mathrm{~mm}$, as illustrated in Fig.

13 4. This results in a model with approximately 100 million nodes and allows for time separation

14 of reflections at the specimen boundaries.

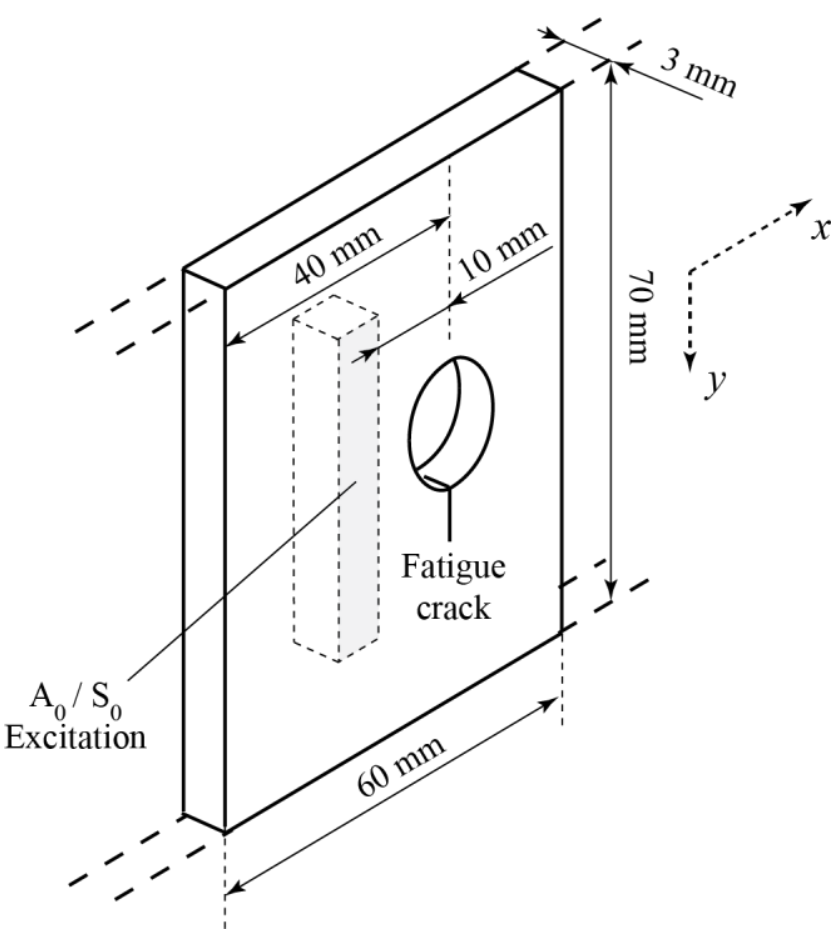

16 Fig. 4: Specimen section implemented in numerical simulation, including fastener hole and excitation position 
1 As the high frequency wave generated in the specimen is a linear superposition of the

2 fundamental Lamb modes $\mathrm{A}_{0}$ and $\mathrm{S}_{0}$ with an energy distribution in the plate thickness

3 depending on the distance from the excitation position, the scattering of the two fundamental

4 modes at the fastener hole was simulated separately. The fundamental modes were generated

5 in the dotted excitation area shown in Fig. 4, $10 \mathrm{~mm}$ from the hole center, by imposing the

6 complete displacement field of the corresponding mode in the two initial simulation time steps.

7 In-plane and out-of-plane displacement components were calculated at every node using FFT

8 as described by Munasinghe [29]. The length ( $x$-direction) of the excitation area was defined

9 as a function of the number of cycles of the generated tone burst multiplied by the Rayleigh

10 wavelength of $1.3 \mathrm{~mm}$ at $2.25 \mathrm{MHz}$. The width (y-direction) was chosen to account for the

11 limited beam spreading from the excitation wedge in the far field. The lateral amplitude

12 distribution of the incident wave was evaluated experimentally and approximated using a

13 polynomial function of second order and applied to the initial displacement field computed as

14 explained above.

15 The predicted time series of the out-of-plane displacement components are saved on both specimen surfaces for a square area $(10 \mathrm{~mm}$ x $10 \mathrm{~mm})$ around the fastener hole. Fast Fourier transform was applied to the recorded time series for each mode. The resulting complex amplitudes (phase and amplitude) of the out-of-plane displacement component were subsequently phase shifted to take into account the additional propagation distance of $115 \mathrm{~mm}$. The phase shift was calculated for every frequency within the signal bandwidth (approx. 1.8 $21 \mathrm{MHz}$ ) as the product of this distance with the corresponding wave number. The high-frequency wave scattering is obtained by combining the $A_{0}$ and $S_{0}$ simulation results. This procedure allows to predict amplitude and phase of the high-frequency guided wave scattered at the

24 fastener hole for any frequency within the excitation bandwidth, in particular at the center 25 frequency of $2.25 \mathrm{MHz}$, for any experimental excitation position along the specimen (neglecting the dependence of the beam spread on the excitation distance). The normalization 
1 procedure based on the amplitude of the incident wave, described in the previous chapter, was

2 applied to the simulation results.

3 As the procedure described in the previous section generates an artificial time delay of the $\mathrm{A}_{0}$

4 and $S_{0}$ pulses when transferred back in the time domain, an additional, negative phase shift

5 (product between angular frequency and averaged propagation time of $\mathrm{A}_{0}$ and $\mathrm{S}_{0}$ for the

6 distance of $115 \mathrm{~mm}$ ), was added to the frequency spectra of both fundamental Lamb modes.

7 The resulting spectra were added together and transformed back into the time domain by means

8 of inverse FFT, leading to time series corresponding to the out-of-plane displacement

9 measurements around the fastener hole.

\section{4. Analysis of the scattered field}

\section{$11 \quad$ 4.1. Scattering at an undamaged fastener hole}

12 Fig. 5a-d shows time snapshots for the simulation of the scattering at the undamaged hole. The

13 incident wave interacts with the hole and quasi-circular Lamb waves (fundamental and higher

14 modes) are scattered at the hole (Fig. $5 b$ ) and propagate radially outwards, interacting with the

15 incident wave pulse. Behind the hole, the wave front curves around the hole generating 16 creeping waves [30] propagating around the circumference of the hole (Fig. 5c/d), and lower

17 amplitude is visible in the shadow area of the hole relative to the propagation direction of the

18 incident wave. As the creeping waves propagate along the stress-free internal face of the hole

19 they permanently radiate energy outwards and their amplitude decreases. Creeping waves are

20 generated symmetrically on both sides of the hole and interfere behind the hole (Fig. $5 d$ ). 

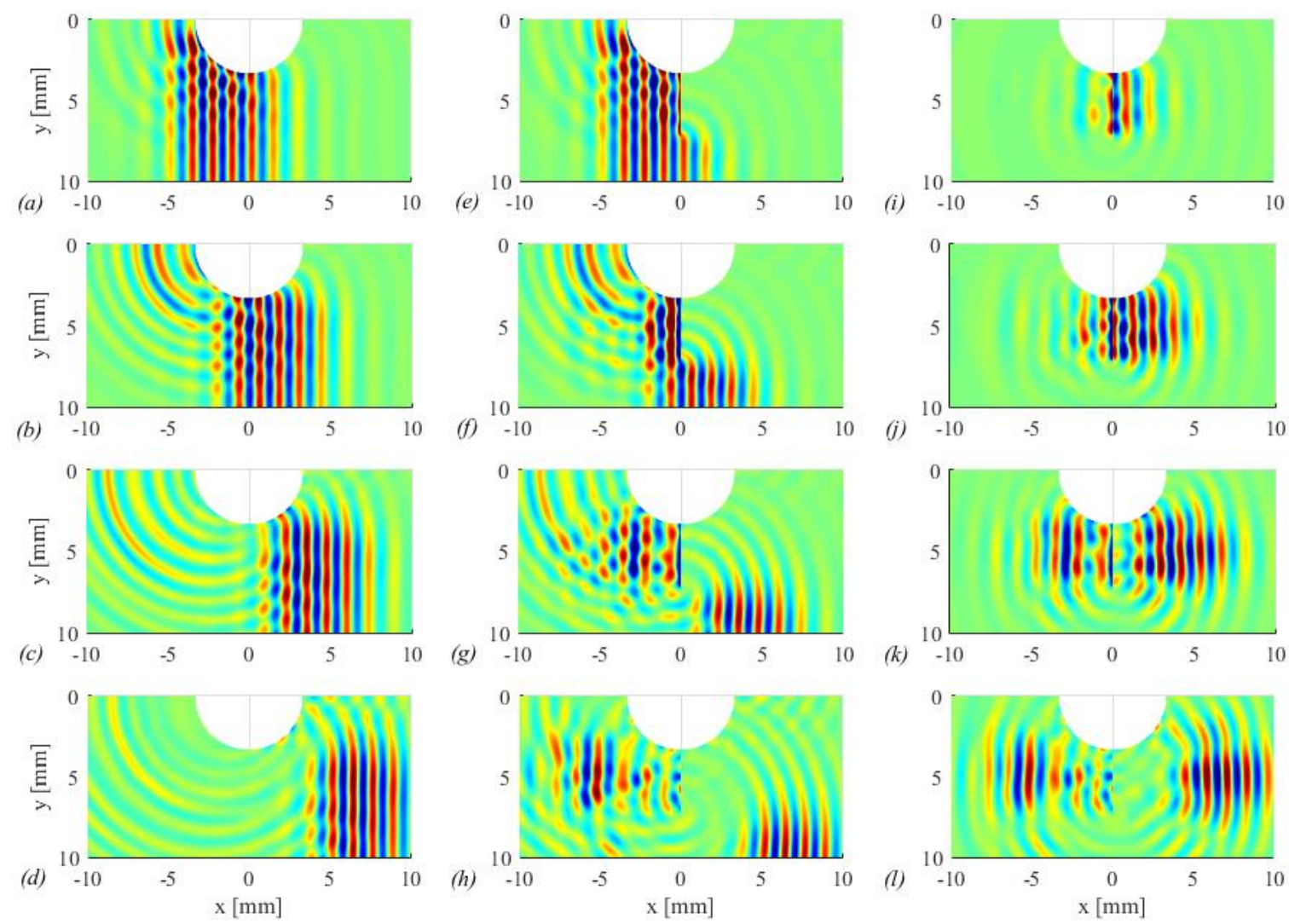

1 Fig. 5: (COLOR) Displacement amplitude snapshots of simulated wave on specimen front side; scattering at undamaged hole (a-d), scattering at $4 \mathrm{~mm}$ through thickness crack (e-h), difference between scattering at hole with $4 \mathrm{~mm}$ through thickness crack and without crack (i-l). Time snapshots at $4.4 \mu \mathrm{s}, 5.4 \mu \mathrm{s}, 6.4 \mu \mathrm{s}$ and $7.4 \mu \mathrm{s}$ from top to bottom line.

6 For both the simulation and the experimental measurement the time trace recorded at each point

7 of the grid was converted to the frequency domain using FFT. The amplitude of the scattered

8 field was extracted at the center frequency and is shown in Fig. 6 for a circular area around the

9 hole. Fig. $6 a$ and $6 b$ represent the measured amplitude for front and back (transducer) side,

10 respectively, with lower amplitude on the back side visible. Selecting the transducer excitation

11 position approximately half a beatlength from the hole leads to an energy concentration on the

12 front side of the specimen [26], where the fatigue crack is expected to grow. The predicted

13 amplitude of the scattered field is shown in Fig. $6 c$ and Fig $6 d$. Comparison with Fig. $6 a$ and

$146 b$ shows a very good agreement, with similar amplitude ratios and patterns. 

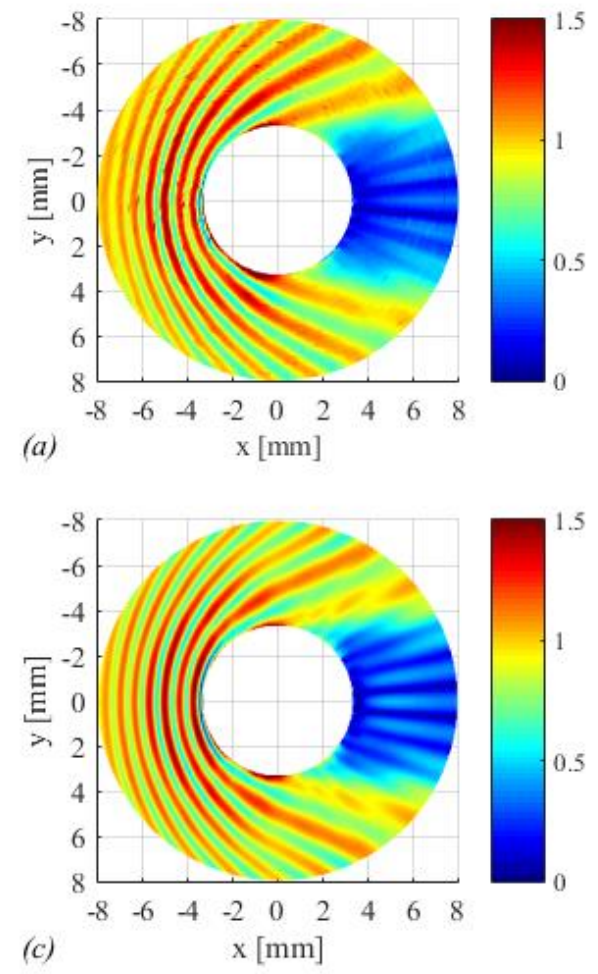
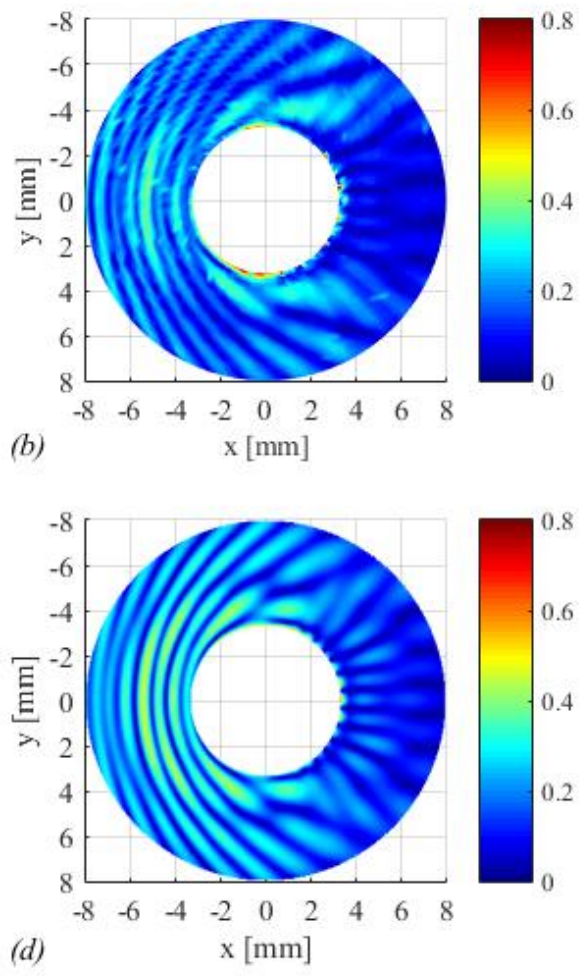

Fig. 6: (COLOR) Normalized amplitude of scattered field at undamaged fastener hole: $f_{0}=2.25 \mathrm{MHz}$;

measurement on front side (a) and back side (b), numerical prediction on front side (c) and back side (d).

5 The area behind the hole (positive $x$ axis) is characterized by low amplitudes, as it is located in

6 the shadow area of the hole. The maxima and minima pattern observable in that area are

7 generated by the interference between the creeping waves. The interference pattern generated

8 by the interaction between the reflected and incident wave is observable on the specimen front

9 side (Fig. $6 a / c$ ) in front of the fastener hole. The amplitude along the negative $x$ axis is shown

10 in Fig. 7 for comparison between the measured and simulated amplitude patterns. It must be

11 noted that the amplitudes are normalized as described in section III, i.e., only relative amplitude

12 variations can be compared. The experimentally measured amplitudes show some scattering,

13 but in general the same pattern of high and low amplitude due to the constructive and

14 destructive interference between the incident and scattered wave can be observed. This helps

15 to validate the simulation procedure proposed in this contribution, simulating $\mathrm{A}_{0}$ and $\mathrm{S}_{0}$

16 scattering separately and combining the signals with the corrected phase difference. 


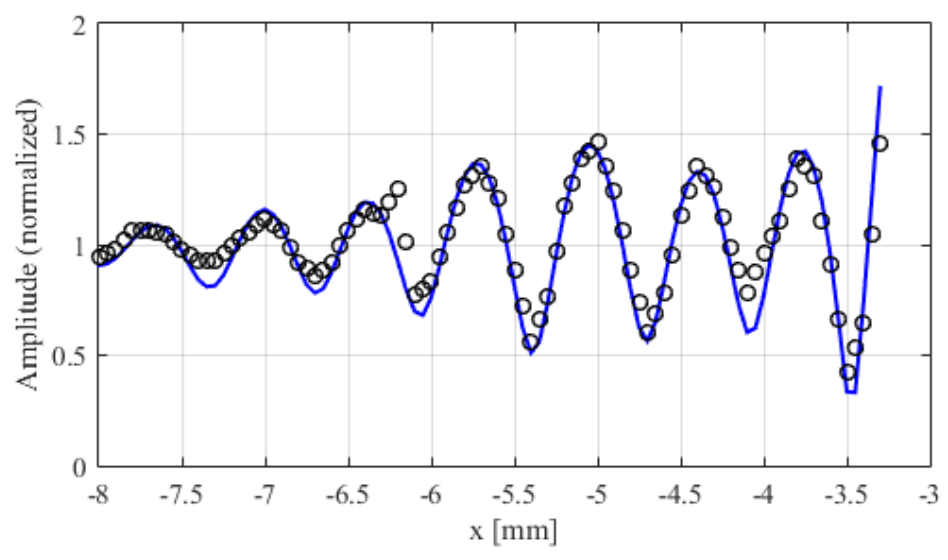

$1 \quad$ Fig. 7: Normalized amplitude of scattered field on specimen center line $(y=0)$, front side, in front of undamaged

2 fastener hole: $f_{0}=2.25 \mathrm{MHz}$; measurement (circles) and simulation (line).

3

4 4.2. Scattering at a fastener hole with a through thickness fatigue crack

5 The simulated scattering at the $4 \mathrm{~mm}$ long through thickness crack at the side of the hole can

6 be seen from the time snapshots in Fig. $5 e-h$. The crack blocks the wave propagation with

7 limited energy behind its location and leads to a strong, additional back-scattered wave. Fig.

$85 f / g$ show a curved wave front behind the defect location with reduced amplitude as compared

9 to Fig. 5b/c. The difference signal between the scattering at the hole with the through thickness

10 crack and only the hole (baseline) has been calculated from the simulation results and the

11 matching time snapshots are shown in Fig. 5i-l. As the crack length is approximately 3 times

12 the wavelength it acts in a first approximation as a line source, with scattered waves with planar

13 wave fronts propagating in the forward and backward directions. The back-scattered wave

14 (propagating in negative $x$ direction) interacts with the incident wave and the wave scattered at

15 the hole, leading to the strong variation in amplitude observed for example in Fig. $5 g$. The

16 forward scattered wave is out-of-phase compared to the incident wave and leads to the observed

17 amplitude reduction behind the crack (e.g. Fig. 5h). It should be noted that the wave scattered

18 at the crack shows amplitude variation in the $y$ direction due to a range of causes including the

19 higher and evanescent wave modes and, behind the crack, the lack of the quasi-circular

20 scattering at the hole and the curved wave propagation past the crack tip. 

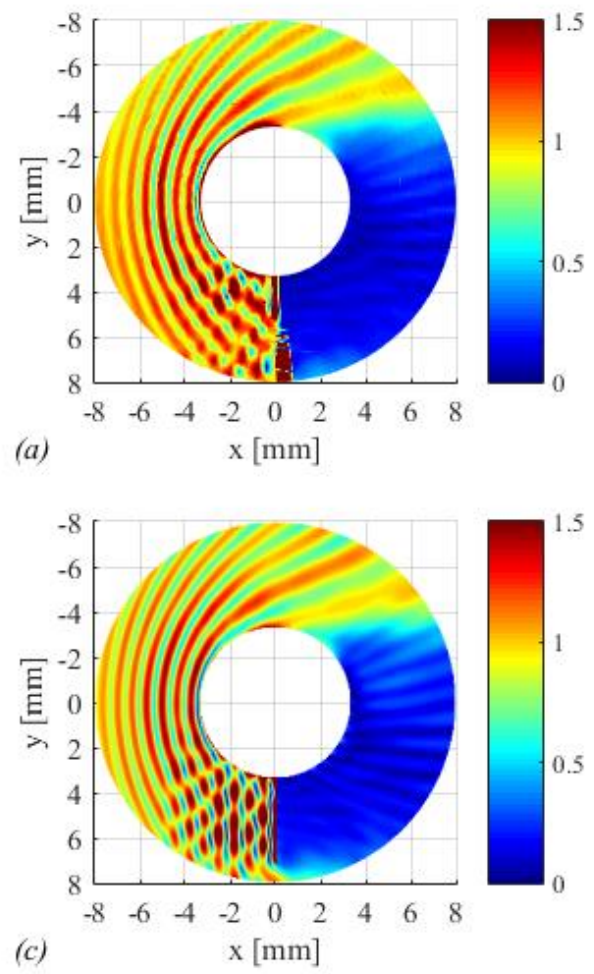
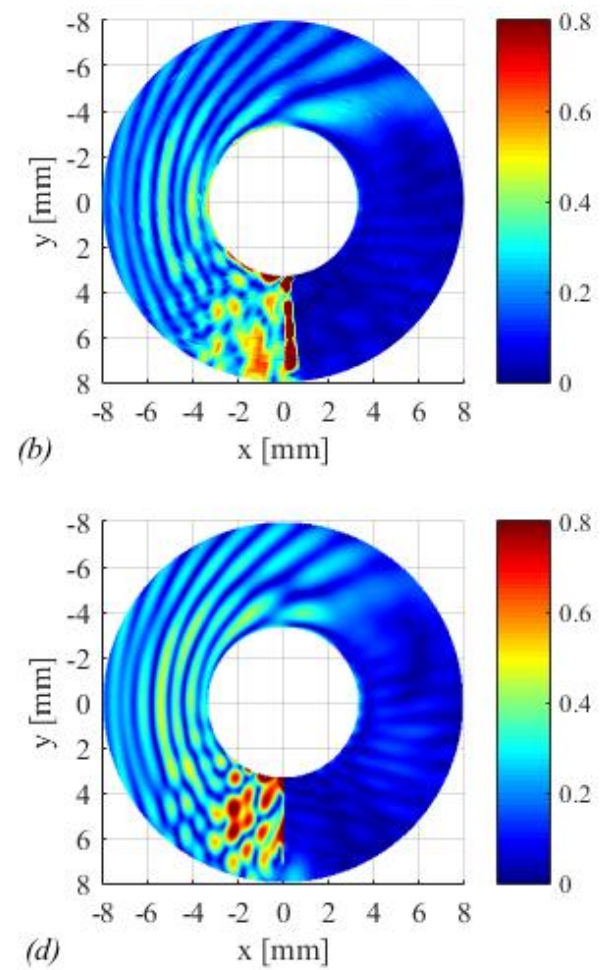

2 Fig. 8: (COLOR) Normalized amplitude of scattered field at fastener hole with $4 \mathrm{~mm}$ through thickness fatigue 3 crack: $f_{0}=2.25 \mathrm{MHz}$; measurement on front side (a) and back side (b), numerical prediction on front side (c) and 4 back side $(d)$.

6 The amplitude of the scattered field around the fastener hole with the $4 \mathrm{~mm}$ through thickness

7 fatigue crack is shown in Fig. 8. Good agreement is obtained between numerical predictions and experiments (compare Fig. $8 a / b$ with Fig. $8 c / d$ ). The differences between simulations and experiments can be primarily related to the difference between the idealized, perfectly plane crack geometry implemented in the numerical code and the real crack geometry with not perfectly straight crack surfaces. It has been shown that small differences in the exact crack location, direction, and shape can lead to significant differences in terms of the reflected pulse, as the interference between the $\mathrm{A}_{0}$ and $\mathrm{S}_{0}$ modes and the reflection angle at the crack surface affect the pulse echo-measurements [11]. The presence of the crack strongly disturbs the lateral symmetry of the scattering pattern at the hole. For both front and back side of the specimen, the through thickness fatigue crack leads to a shadow area behind the crack where very little 
1 energy arrives. The interaction of the additional Lamb waves propagating back towards the 2 excitation source with the incident wave field and the waves scattered at the hole leads to an 3 additional, complicated interference pattern in front of the crack that can be observed on both 4 specimen sides. The Lamb modes reflected by the crack propagate back towards the excitation 5 location and can be used in a pulse-echo configuration to monitor fatigue crack growth. Pulse6 echo time traces presented in a previous contribution have shown a significant, reflected pulse 7 [11]. The presence of the crack also affects the interference pattern generated by the creeping 8 waves right behind the hole. The creeping wave generated on the damaged side of the hole is 9 phase shifted and its amplitude is strongly reduced by the presence of the crack. This results in 10 a clear decay and shift of the interference pattern compared to the pattern behind the 11 undamaged hole.

12 The predicted scattered field at the undamaged hole (baseline signal) was subtracted from the 13 scattered field at the hole with the through thickness crack, allowing for the separation of the waves reflected at the crack from the direct reflection at the hole. This is equivalent to taking the complex difference (amplitude and phase information) between the scattered fields, shown in Fig. $9 a$ for the $4 \mathrm{~mm}$ through thickness crack. The scattering due to the crack can be observed clearly, with higher amplitude in the forward scattered direction (positive $x$ axis) than in the back scattered direction (negative $x$ axis). Due to the multiple interferences of different modes and the shape of the wave fronts described previously, a clear pattern is visible with higher and lower difference at varying locations before and behind the crack. 

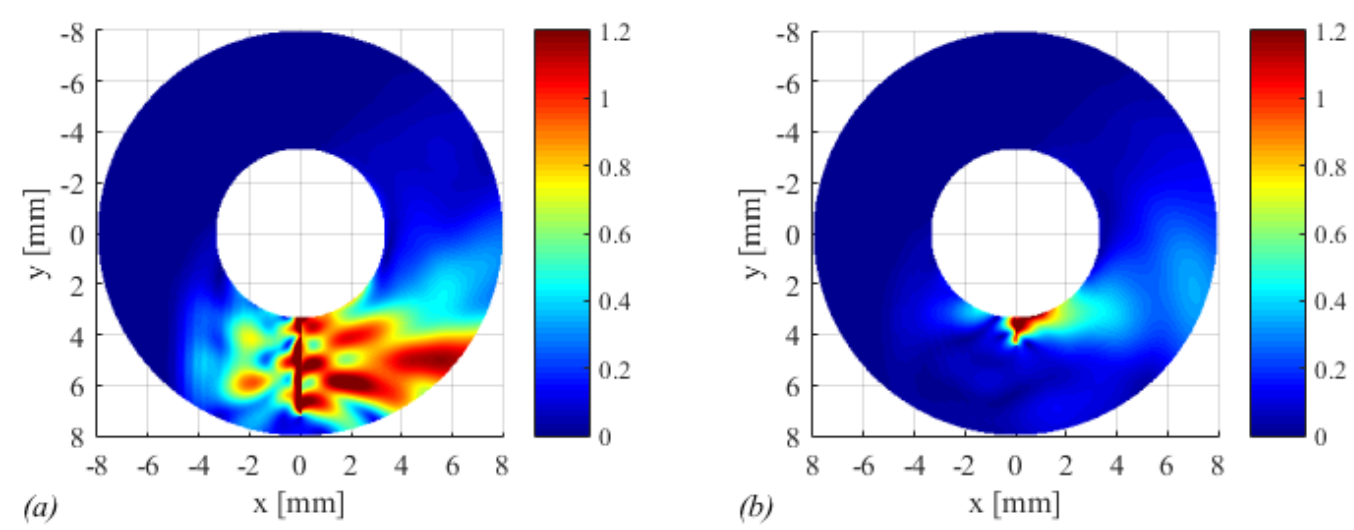

2

3

Fig. 9: (COLOR) Predicted amplitude of complex difference between scattered field at fastener hole with and without crack, front side of the specimen. $4 \mathrm{~mm}$ through thickness crack (a) and $1.2 \mathrm{~mm}$ quarter-elliptical crack (b).

7 4.3. Scattering at a fastener hole with a part-thickness fatigue crack

8 The measured scattered field at a fastener hole with a $1.2 \mathrm{~mm}$ quarter-elliptical fatigue crack is

9 illustrated in Fig. 10a and Fig. 10b. The crack length and depth are approximately equal to the wavelength of the incident high-frequency guided wave with a crack area of approximately 1 $\mathrm{mm}^{2}$. The presence of the crack affects the lateral symmetry characteristics of the scattered field, but due to the smaller crack size a smaller effect compared to Fig. 8 is observed. The incident wave is partly stopped by the crack. On the front (damaged) side of the specimen, this generates a shadow area behind the crack where little energy arrives and a modification of the scattering pattern as compared to the undamaged hole. As the damage is small (size comparable to the wavelength) and only partially through the thickness, on the back side of the specimen a change in the scattered field is visible, but no significant drop in amplitude.

18 The prediction from the numerical simulations matches well with the measured amplitude

19 fields (compare Fig. 10a/b and Fig. 10c/d). All characteristic features described above can be observed in the numerical predictions. In a previous contribution, it had been shown that, for 
1 cracks typically of the same order of magnitude or smaller than the wavelength of the incident

2 high-frequency guided wave, the noncontact measurement of the out-of-place displacement

3 component behind the crack on the front side of the specimen can be used for fatigue crack

4 growth monitoring [28]. The measurement point selected in that work was located in the

5 shadow area behind the crack, where the incident wave is partly reduced by the presence of the

6 crack. This can be seen from the complex difference compared to the baseline case (no crack)

7 shown in Fig. 9b. The largest difference is predicted to be located just behind the damage

8 location close to the fastener hole. The radiation of the waves scattered at the part-thickness

9 crack can be observed with larger amplitude for the forward scattered wave leading to the amplitude drop behind the crack. Compared to the considered larger, through-thickness crack, the scattered field for a defect size comparable to the wavelength is more uniform as less interference between the different additional scattered wave modes occurs.
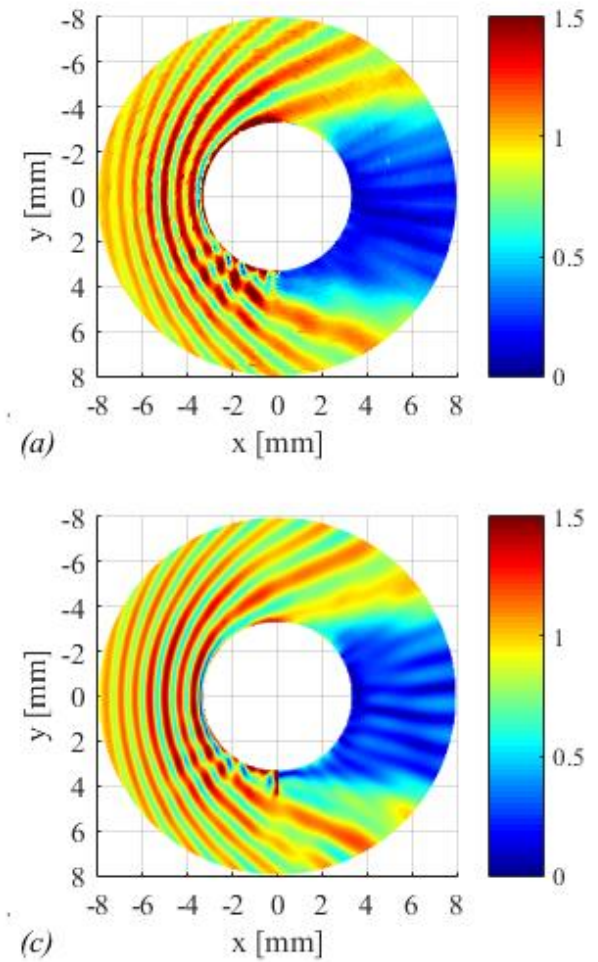
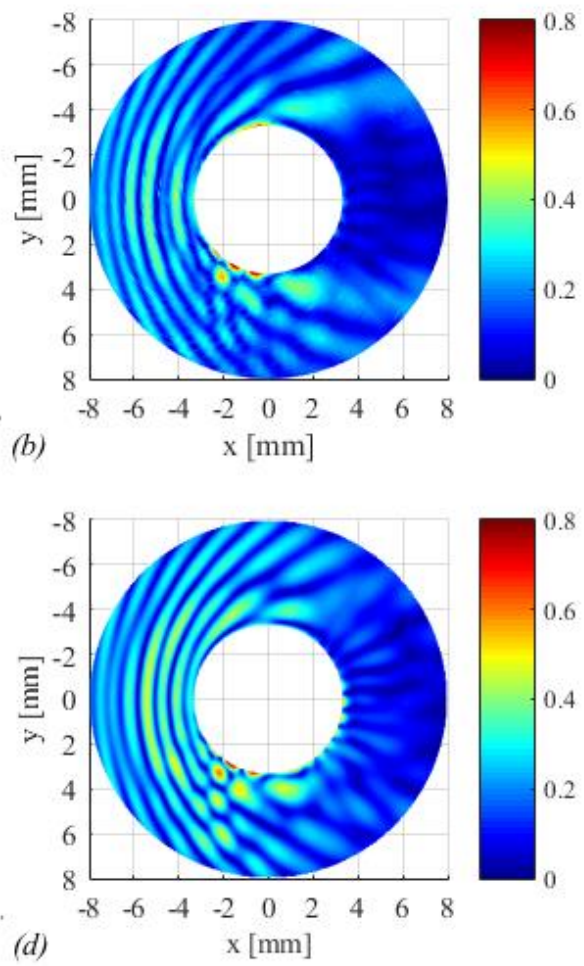

14 Fig. 10: (COLOR) Normalized amplitude of scattered field at fastener hole with $1.2 \mathrm{~mm}$ quarter-elliptical fatigue crack: $f_{0}=2.25 \mathrm{MHz}$; measurement on front side (a) and back side (b), numerical prediction on front side (c) and 
1 For comparison the amplitude for the three considered cases is shown in Fig. 11 on a line 2 perpendicular to the incident wave direction, $1 \mathrm{~mm}$ behind the crack location. For the baseline 3 case (no crack, hole only), the simulated scattered amplitude remains symmetric. The measured 4 amplitude (Fig. 11a) shows some minor variations, but overall the expected symmetry. From 5 the simulation results shown in Fig. $11 b$ one can see that on the hole side opposite to the crack 6 location almost no change in the scattering pattern is predicted. The experimental 7 measurements on that side (Fig. 11a) show the same pattern but with some variation (mostly 8 in amplitude) as the specimen and transducer were removed for the fatigue crack growth 9 cycling. This gives a good indication of the repeatability of the high frequency guided wave 10 measurements. For the small quarter-elliptical fatigue crack both the measurement and 11 simulation show the observed amplitude drop close to the hole, but with a slightly different 12 amplitude pattern. The cause for this is most likely the fact that the experimental fatigue crack 13 differs slightly in size, location and orientation from the modeled, regular geometry, which has 14 been shown to lead to variations in the interference between the different scattered wave modes. 15 For the large, through thickness fatigue crack the measured and predicted amplitude pattern match very well, showing the significant drop in amplitude behind the damage location. 

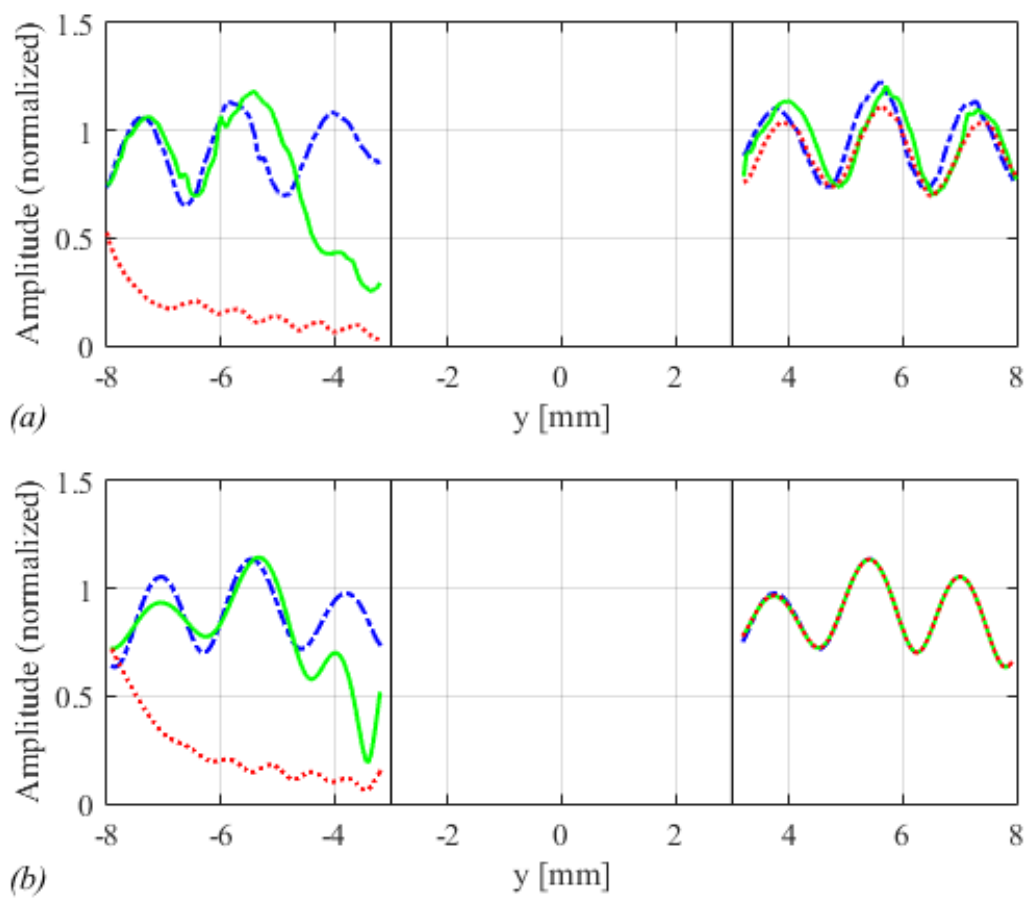

$1 \quad$ Fig. 11: Normalized amplitude of scattered field on line perpendicular to wave propagation direction located 1

$2 \mathrm{~mm}$ behind crack position $(x=1 \mathrm{~mm}), f_{0}=2.25 \mathrm{MHz}$; undamaged fastener hole (dashed line), fastener hole with

$31.2 \mathrm{~mm}$ quarter-elliptical fatigue crack (solid line) and fastener hole with $4 \mathrm{~mm}$ through thickness crack (dotted

4 line); measurement (a) and simulation (b). Hole boundaries delimited by vertical lines.

5

6 5. Conclusions

7 The scattering of high frequency guided waves by a fatigue crack at the side of a fastener hole

8 has been studied. The specific type of high frequency guided waves employed consists of a

9 superposition of the two fundamental modes $\mathrm{A}_{0}$ and $\mathrm{S}_{0}$ at about $6.75 \mathrm{MHz} \mathrm{mm}$, where there is

10 a small difference in the respective phase velocities. This leads to an energy exchange between

11 the plate sides over the beatlength and can be used to improve the sensitivity for the detection

12 of small part-thickness fatigue cracks originating on one surface of an aircraft structure. The

13 scattered field around an undamaged, circular hole, for a quarter-elliptical part-thickness, and

14 longer through-thickness fatigue crack grown at the side of the hole were measured. The

15 scattered field was simulated using a 3D finite difference algorithm, with an analytical model 
1 to correct for the complete propagation distance from the wedge transducer to the hole, so that

2 only a limited area of interest around the hole had to be simulated. Good agreement between

3 the full field laser measurements and the finite difference simulations was found. The complex

4 difference of the scattered field due to the fatigue cracks was evaluated. For the small, part-

5 thickness crack the largest difference was found on the damaged side behind the crack location

6 due to the blocking of the incident wave leading to a shadow area with only a smaller reflected

7 (back-scattered) wave pulse. For the larger, through-thickness crack larger magnitude of the

8 forward and backward scattered wave pulses with significantly reduced amplitude on both plate

9 sides behind the crack and a large back-scattered wave pulse were observed. These evaluations

10 can be used to predict the sensitivity of nondestructive and structural health monitoring

11 systems, e.g. to predict the sensitivity for the detection of such fatigue cracks from local point

12 laser monitoring measurements sensitive for small cracks and pulse-echo measurements from

13 a stand-off distance which have been shown to be sensitive for longer, through-thickness

14 fatigue cracks. 
2 [1] J. M. Papazian, J. Nardiello, R. P. Silberstein, G. Welsh, D. Grundy, C. Craven, L. Evans, N. Goldfine, J. E. Michaels, T. E. Michaels, Y. F. Li, and C. Laird, Sensors for monitoring early stage fatigue cracking, Int. J. Fatigue 29, 1668-1680 (2007).

[2] A. C. Cobb, J. E. Michaels, and T. E. Michaels, An automated time-frequency approach for ultrasonic monitoring of fastener hole cracks, NDT \& E Int. 40, 525-536 (2007).

[3] B. Mi, J. E. Michaels, and T. E. Michaels, An ultrasonic method for dynamic monitoring of fatigue crack initiation and growth, J. Acoust. Soc. Am. 119, 74-85 (2006).

[4] P. D. Wilcox, M. J. S. Lowe, and P. Cawley, Mode and transducer selection for long range Lamb wave inspection, J. Intell. Mater. Syst. Struct. 12, 553-565 (2001).

[5] Y.-H. Pao and C. C. Chao, Diffractions of flexural waves by a cavity in an elastic plate, AIAA J. 2, 2004-2010 (1964).

[6] P. Fromme and M. B. Sayir, Measurement of the scattering of a Lamb wave by a through hole in a plate, J. Acoust. Soc. Am. 111, 1165-1170 (2002).

[7] J. C. P. McKeon and M. K. Hinders, Lamb wave scattering from a through hole, J. Sound Vibration 224, 843-862 (1999).

[8] P. Fromme and M. B. Sayir, Detection of cracks at rivet holes using guided waves, Ultrasonics 40, 199-203 (2002).

[9] C. Doherty and W. K. Chiu, Scattering of ultrasonic-guided waves for health monitoring of fuel weep holes, Struct. Health Monit. 11, 27-42 (2012).

[10] H. Cho and C. J. Lissenden, Structural health monitoring of fatigue crack growth in plate structures with ultrasonic guided waves, Struct. Health Monit. 11, 393-404 (2012). 
1 [11] B. Masserey and P. Fromme, In-situ monitoring of fatigue crack growth using high frequency guided waves, NDT \& E Int. 71, 1-7 (2015).

[12] N. Terrien, D. Osmont, D. Royer, F. Lepoutre, and A. Déom, A combined finite element and modal decomposition method to study the interaction of Lamb modes with micro-defects, Ultrasonics 46, 47-78 (2007).

[13] D. W. Greve, P. Zheng, and I. J. Oppenheim, The transition from Lamb waves to longitudinal waves in plates, Smart Mater. Struct. 17, 035029 (2008).

[14] B. W. Ti, W. D. O’Brien, and J.G. Harris, Measurements of coupled Rayleigh wave propagation in an elastic plate, J. Acoust. Soc. Am. 102, 1528-1531 (1997).

[15] I. A. Viktorov, Rayleigh and Lamb waves (Plenum, New York, 1967), pp. 93-96.

[16] R. Paskaramoorthy, A. H. Shah, and S. K. Datta, Scattering of flexural waves by cavities in a plate, Int. J. Solids Struct. 25, 1177-1191 (1989).

[17] Z. Chang and A. Mal, Scattering of Lamb waves from a rivet hole with edge cracks, Mech. Mater. 31, 197-204 (1999).

[18] Y. Cho and J. L. Rose, A boundary element solution for a mode conversion study on the edge reflection of Lamb waves, J. Acoust. Soc. Am. 99, 2097-2109 (1996).

[19] A. H. Harker, Numerical modelling of the scattering of elastic waves in plates, J. Nondestruct. Evaluat. 4, 89-106 (1984).

[20] M. Hirao, H. Fukuoka, and Y. Miura, Scattering of Rayleigh surface waves by edge cracks: Numerical simulation and experiment, J. Acoust. Soc. Am. 72, 602-606 (1982).

[21] J. Virieux, P-SV wave propagation in heterogeneous media: Velocity-stress finitedifference method, Geophysics 51, 889-901 (1986).

[22] R. Madariaga, Dynamics of an expanding circular fault, Bull. Seismol. Soc. Am. 66, 639-666 (1976). 
1 [23] B. Masserey and E. Mazza, Analysis of the near-field ultrasonic scattering at a surface crack, J. Acoust. Soc. Am. 118, 3585-3594 (2005).

[24] B. Masserey, L. Aebi, and E. Mazza, Ultrasonic surface crack characterization on complex geometries using surface waves, Ultrasonics 44, e957-e961 (2006).

[25] P. Fellinger, R. Marklein, K. J. Langenberg, and S. Klaholz, Numerical modeling of elastic wave propagation and scattering with EFIT-elastodynamic finite integration technique, Wave Motion 21, 47-66 (1995).

[26] B. Masserey and P. Fromme, On the reflection of coupled Rayleigh-like waves at surface defects in plates, J. Acoust. Soc. Am. 123, 88-98 (2008).

[27] B. Masserey and P. Fromme, P. In-situ Monitoring of Fatigue Crack Growth at Fastener Holes Using Rayleigh-like Waves, in Rev. of Prog. in QNDE 27B, ed. by D.O. Thompson and D.E. Chimenti, AIP Conference Proceedings 975, New York, 14841491 (2008).

[28] B. Masserey and P. Fromme, Fatigue Crack Growth Monitoring using High Frequency Guided Waves, Struct. Health Monit. 12, 484-493 (2013).

[29] M. Munasinghe and G. W. Farnell, Finite difference analysis of Rayleigh wave scattering at vertical discontinuities, J. Geophys. Res. 78, 2454-2466 (1973).

[30] W. Hassan and P. Nagy, Circumferential creeping waves around a fluid-filled cylindrical cavity in an elastic medium, J. Acoust. Soc. Am. 101, 2496-2503 (1997). 